\title{
EFEITO DA RESTRIÇÃO ALIMENTAR DURANTE O FINAL DA GESTAÇÃO SOBRE O PEŞO AO NASCER DE CORDEIROS SANTA INÊS ${ }^{1}$
}

\author{
Effects of maternal undernutrition during final pregnancy on weight birth of Santa Ines lambs
}

\author{
Luciana Castro Geraseev², Juan Ramon Olalquiaga Perez ${ }^{3}$, Rodrigo Palomo de Oliveira ${ }^{4}$, \\ Fábio Arantes Quintão ${ }^{4}$, Bruno Carneiro e Pedreira ${ }^{5}$
}

\begin{abstract}
RESUMO
O experimento foi conduzido no setor de Ovinocultura da Universidade Federal de Lavras, com o objetivo de avaliar o peso ao nascer de cordeiros da raça Santa Inês, machos e fêmeas, nascidos de ovelhas submetidas ou não a uma restrição alimentar durante o terço final da gestação. Para tanto, foram utilizadas 44 ovelhas Santa Inês gestando gêmeos, as quais com 100 dias de gestação, foram confinadas e divididas em dois grupos: um grupo recebeu alimentação à vontade para satisfazer suas necessidades energéticas, e o segundo grupo recebeu alimentação restrita para satisfazer $60 \%$ das suas necessidades energéticas. O cálculo das necessidades energéticas foi baseado nas recomendações do ARC (1980), levando-se em consideração o peso da ovelha, o tempo de gestação e o número de fetos que cada ovelha estava gestando. Com relação as ovelhas, foram avaliados o peso corporal no início do experimento e logo após a parição, a duração da gestação e a massa biológica produzida. Com relação aos cordeiros, foram avaliados o peso ao nascer dos mesmos, sendo que para tanto estes animais foram divididos de acordo com o sexo (machos e fêmeas). O peso ao nascer dos cordeiros machos e fêmeas foi afetado pela restrição pré-natal, sendo que a média do peso ao nascer dos cordeiros machos sem restrição foi 4,162 kg e com restrição $2,893 \mathrm{~kg}$; e o peso ao nascer das cordeiras fêmeas sem restrição foi $3,474 \mathrm{~kg}$ e com restrição 2,855 $\mathrm{kg}$. A grande redução observada neste trabalho no peso ao nascer dos cordeiros machos $(30,5 \%)$ e fêmeas $(17,8 \%)$ revela a importância da adoção de um nível nutricional adequado para as ovelhas gestantes, principalmente durante o terço final da gestação.
\end{abstract}

Termos para indexação: Gestação, ovinos, restrição alimentar.

\begin{abstract}
An experiment was conducted in the Sheep Production Sector of the Animal Science Department at the Federal University of Lavras, Lavras - MG to evaluate weight birth of Santa Ines lambs, males and females from ewes submitted or not to feed restriction during final pregnancy. A total of 44 ewes Santa Ines with twin lambs were utilized , divided in two groups: the first group was ad libitum fed and other was restricted fed (60\% of energy requirements) during final pregnancy. The nutritional requirements for pregnancy ewes were calculated by ARC (1980) recommended with ewe's weight, gestation length and foetuses number. For ewes were evaluated initial and post-partum weight, gestation length and biological mass. For lambs were evaluated birth weight of males and females. The birth weight of males and females were affected by prenatal restriction, and the overall weight birth of males without restriction were $4.162 \mathrm{~kg}$ and with restriction were $2.893 \mathrm{~kg}$; the overall weight birth of females without restriction were $3.474 \mathrm{~kg}$ and with restriction were $2.855 \mathrm{~kg}$. The prenatal restriction reduced birth weight of males in $30.5 \%$ and $17.8 \%$ of females.
\end{abstract}

Index terms: Feed restriction, pregnancy, sheep.

(Recebido para publicação em 1 de outubro de 2003 e aprovado em 10 de outubro de 2005)

\section{INTRODUÇÃO}

O peso ao nascer, como consequência do crescimento fetal, está diretamente relacionado com a mortalidade perinatal em ovinos e bovinos, quando pesos ao nascer muito abaixo da média reduzem a taxa de sobrevivência de cordeiros, afetando dessa forma a produtividade do sistema de produção (ROBINSON, 1982).

Vários fatores podem influenciar o crescimento fetal, entre os quais podemos destacar: o nível de nutrição materna, sexo do cordeiro, tipo de gestação (simples ou múltipla), raça dos pais, idade da mãe, etc.

Entre esses fatores destaca-se a influência do nível nutricional da mãe, principlamente durante o terço final da gestação, pois é neste período que ocorre o maior desenvolvimento do feto, acarretando em uma grande demanda de nutrientes pelo mesmo. Segundo Ferrel (1992), o crescimento fetal é o resultado de um balanço entre o potencial genético para o crescimento e os limites impostos no suprimento dos nutrientes. Assim, restrições nutricionais severas impostas às mães, principalmente durante o terço final da gestação, afetam marcadamente o peso ao nascer dos cordeiros.

\footnotetext{
${ }^{1}$ Extraído da Tese de Doutorado apresentada pelo primeiro autor à Universidade Federal de Lavras.

${ }^{2}$ Professora da Universidade Federal de Minas Gerais/Campus Montes Claros - Cx. P. 135 - Av.Osmane Barbosa, s/n Montes Claros, MG Igeraseev@ufmg.br

${ }^{3}$ Professor da Universidade Federal de Lavras/UFLA - Cx. P. 3037 - 37.200-000 - Lavras, MG - roperez@ufla.br

${ }^{4}$ Alunos de Pós-Graduação da Universidade Federal de Lavras - Cx. P. 3037 - 37.200-000 - Lavras, MG

${ }^{5}$ Aluno de Pós-Graduação da Escola Superior de Agronomia Luiz de Queiroz - brunopedreira@uol.com.br
} 
Segundo Grant \& Helferich (1991), o crescimento pré-natal é rápido, ocorrendo a uma taxa exponencial em todas as espécies animais. No início da gestação o crescimento do feto é pequeno sendo regido por padrões genéticos da espécie, já no terço final da gestação ocorre um grande crescimento do feto, sendo este altamente influenciado pela nutrição materna. Segundo Ferrel (1992), 90\% do peso ao nascer de ovinos é obtido durante os últimos $40 \%$ da gestação.

Mellor (1987) afirma que o padrão de crescimento fetal é afetado pelo plano de nutrição materno no estágio final da gestação. De acordo com o autor, quando fêmeas bem alimentadas sofrem uma restrição severa e abrupta, a taxa de crescimento fetal pode decrescer até $40 \%$, sendo que as perdas são ainda maiores quando a restrição prossegue por mais de duas semanas.

Widdowson \& Lister (1991) explicam que algumas substâncias como, oxigênio, dióxido de carbono e água são difundidas livremente na circulação placentária, entretanto, substâncias como a glicose (principal fonte energética durante a vida intra-uterina) é difundida passivamente por meio de carreadores moleculares específicos. Dessa forma, restrições severas impostas à mãe, principalmente em espécies multíparas, podem levar a uma redução na concentração de glicose afetando a velocidade de difusão desta por meio da circulação placentária, com consequiências no suprimento energético do feto.

A restrição no período pré-natal tem conseqüências no metabolismo do animal também na fase pós-natal. Greenwood et al. (1998) afirmam que o metabolismo energético do animal é afetado principalmente nas primeiras semanas de vida. De acordo com os autores, animais submetidos à restrição alimentar pré-natal possuem uma capacidade limitada de utilização da energia para deposição de tecidos, o que resulta em menor crescimento destes animais na fase pós-natal.

Animais submetidos à restrição pré-natal apresentam menores exigências energéticas de mantença e, quando suplementados adequadamente no período pósnatal apresentam uma maior taxa de deposição de gordura na carcaça quando comparados com animais que não sofreram qualquer tipo de restrição (GREENWOOD et al., 1998). De acordo com os autores, esta maior taxa de deposição do tecido adiposo, pode ser devido à capacidade limitada dos tecidos magros (ossos e músculos) em responderem a suplementação.

Objetivou-se com presente trabalho, avaliar os efeitos da restrição pré-natal sobre o peso ao nascer de cordeiros, machos e fêmeas, da raça Santa Inês.

\section{MATERIAL E MÉTODOS}

O trabalho foi realizado no Setor de Ovinocultura do Departamento de Zootecnia da Universidade Federal de Lavras, MG. Utilizaram-se 100 ovelhas Santa Inês, as quais foram cruzadas com um macho da raça Santa Inês. Nessas ovelhas foi feito um tratamento para estimular a ovulação e depois feita a sincronização do cio em grupos de 10 ovelhas por dia. O cruzamento foi feito por meio de monta dirigida para que ocorresse o controle do estágio de gestação das ovelhas. Foi realizado um exame de ultrasonografia nas ovelhas com aproximadamente 60 dias de gestação para identificação do número de fetos que cada ovelha estava gestando, sendo então selecionadas 44 ovelhas gestando gêmeos.

Com 100 dias de gestação as ovelhas foram confinadas e divididas em dois grupos de 22 ovelhas: um grupo recebeu alimentação à vontade para satisfazer suas necessidades energéticas, e o segundo grupo recebeu alimentação restrita para satisfazer $60 \%$ das suas necessidades energéticas. $\mathrm{O}$ cálculo das necessidades energéticas foi baseado nas recomendações do ARC (1980), levando-se em consideração o peso da ovelha, o tempo de gestação e o número de fetos que cada ovelha estava gestando. A composição da dieta total e do concentrado oferecidos às ovelhas encontram-se nas Tabelas 1 e 2.

TABELA 1 - Composição química bromatológica e participação centesimal (\% MS) dos ingredientes da dieta total das ovelhas ${ }^{1}$.

\begin{tabular}{lcccccc}
\hline Ingredientes & MS (\%) & $\mathbf{E M}^{2}(\mathbf{k c a l} / \mathbf{k g})$ & PB (\%) & FDN $(\%)$ & Ca (\%) & P (\%) \\
\hline Cana & 50,0 & & 0,75 & 26,25 & - & 0,020 \\
Polpa Cítrica & 20,0 & & 1,33 & 3,80 & 0,400 & 0,037 \\
Concentrado & 30,0 & & 10,0 & 5,21 & 0,380 & 0,325 \\
Total & 100,0 & 2.279 & 12,08 & 35,26 & 0,780 & 0,383 \\
\hline
\end{tabular}

${ }^{1}$ Análises realizadas no Laboratório de Pesquisa Animal do Departamento de Zootecnia da Universidade Federal de Lavras (UFLA).

${ }^{2}$ Valor obtido mediante a ensaio de digestibilidade conduzido paralelamente. 
TABELA 2 - Composição química bromatológica e participação centesimal (\% MS) dos ingredientes do concentrado das ovelhas ${ }^{1}$.

\begin{tabular}{lccccc}
\hline Ingredientes & MS (\%) & PB (\%) & FDN (\%) & Ca (\%) & P (\%) \\
\hline Farelo de soja & 40,0 & 20,32 & 7,84 & 0,173 & 0,313 \\
Milho grão & 45,0 & 4,36 & 9,52 & 0,013 & 0,140 \\
Uréia & 3,5 & 8,67 & - & - & - \\
Sal Comum & 3,0 & - & - & - & - \\
Super Simples & 3,0 & - & - & - & 0,603 \\
Calcário & 3,5 & - & - & 1,08 & - \\
Sup.Mineral & 2,0 & - & - & - & - \\
Total & 100,0 & 33,35 & 17,36 & 1,267 & 1,057 \\
\hline
\end{tabular}

${ }^{1}$ Análises realizadas no Laboratório de Pesquisa Animal do Departamento de Zootecnia da Universidade Federal de Lavras (UFLA).

Com relação as fêmeas, foram avaliados o peso corporal das ovelhas submetidas ou não à restrição alimentar no início do experimento (cem dias de gestação) e logo após a parição, foram também avaliados a duração da gestação e a massa biológica produzida. A massa biológica produzida correspondeu ao peso total dos cordeiros produzidos pela fêmea em relação ao peso corporal da mãe.

Com relação aos cordeiros, foram avaliados o peso ao nascer dos mesmos, sendo que para tanto estes animais foram divididos de acordo com o sexo (machos e fêmeas). Assim, os tratamentos foram compostos por quatro grupos de cordeiros, um grupo de cordeiros machos que sofreu restrição pré-natal, outro grupo de cordeiros machos que não sofreram restrição, um grupo de cordeiros fêmeas que sofreu restrição pré-natal e um grupo de cordeiros fêmeas que não sofreu restrição.

Para a análise do peso ao nascer foi utilizado o delineamento experimental inteiramente casualizado, com o seguinte modelo matemático:

Sendo:

$$
Y_{i j K}=\mu+\alpha_{i}+t_{j}+(\alpha t)_{i j}+\xi_{i j k}
$$

$\mathrm{Y}_{\mathrm{ijK}} \mathrm{o}$ valor observado do peso ao nascer do cordeiro do tratamento $\mathrm{i}(\mathrm{i}=1,2)$, do sexo $\mathrm{j}(\mathrm{j}=1,2)$;

$\mu$ a média geral;

$\alpha_{i}$ o efeito do nível i de restrição pré-natal (com ou sem restrição) com $\mathrm{i}=1,2$;

$t_{\mathrm{j}}$ o efeito do sexo (macho ou fêmea) $\operatorname{com} \mathrm{j}=1,2$; $(\alpha t)_{i j} o$ efeito da interação restrição $x$ sexo;

$\xi_{\mathrm{ijk}}$ o erro experimental associado a observação $\mathrm{Y}_{\mathrm{ijk}}$, que por hipótese tem distribuição normal com média zero e variância de $\mathrm{s}^{2}$.

\section{RESULTADOS E DISCUSSÃO}

O efeito da restrição alimentar sobre o peso corporal das ovelhas está demonstrado na Tabela 3. Aos cem dias de gestação não existia diferença entre o peso corporal das ovelhas dos dois grupos, entretanto, logo após a parição as ovelhas submetidas à restrição alimentar apresentaram um menor peso corporal comparadas com as ovelhas que não sofreram restrição, o que evidência o uso das reservas corporais destas ovelhas.

De acordo com Charismiadou et al. (2000), a restrição alimentar durante a gestação causa mudanças consideráveis no metabolismo das ovelhas. Segundo os autores, a partição dos nutrientes favorece o crescimento fetal em detrimento aos requerimentos das fêmeas gestantes, dessa forma, animais submetidos a um nível nutricional inadequado durante o terço final da gestação utilizam suas reservas corporais numa tentativa de manter $\mathrm{o}$ aporte de nutrientes adequados ao crescimento fetal, o que resulta em perda de peso das fêmeas.

Sibanda et al. (1999), trabalhando com cabras da raça Matebele recebendo dietas com diferentes níveis nutricionais durante o terço final da gestação, observaram que os animais submetidos a uma restrição de $60 \%$ das recomendações do ARC (1984) apresentaram menores pesos e scores corporais em comparação aos animais sem restrição.

Quanto ao tempo de gestação não houve efeito da restrição nutricional sobre esta variável, sendo o tempo de gestação médio das ovelhas submetidas à restrição nutricional de 148 dias e das sem restrição 145 dias. Estes resultados estão de acordo com os observados por Sibanda et al. (1999), entretanto, diferem dos encontrados por Ivey et al. (2000), que constataram uma redução no tempo de gestação das fêmeas submetidas à restrição alimentar durante o terço final da gestação. 
Na Tabela 4, são apresentadas as médias de peso ao nascer dos cordeiros machos e fêmeas com e sem restrição alimentar pré-natal. Os valores médios encontrados para os cordeiros sem restrição $(3,830 \mathrm{~kg})$ foram superiores aos encontrados por Girão et al. (1999) que ao utilizar cordeiros Santa Inês observou valores médios de 3,39 kg para esta variável.

De acordo com os dados apresentados na Tabela 3, verifica-se que os pesos ao nascer tanto dos machos quanto das fêmeas sem restrição foram superiores ao grupo de animais da restrição pré-natal, o que comprova o comprometimento do desenvolvimento destes últimos durante a gestação. Diversos trabalhos demonstraram a redução do peso ao nascer em cordeiros oriundos de ovelhas submetidas à restrição de energia ou proteína durante a metade e/ou final da gestação (HOLST et al., 1986; ROBINSON, 1982; SIBBALD \& DAVIDSON, 1998; SILVEIRA et al., 1992).
De acordo com Ferrel (1992), o peso ao nascer dos cordeiros pode ser reduzido em média $30 \%$ em conseqüência da restrição alimentar imposta as ovelhas durante o terço final da gestação. No presente trabalho, foi encontrada uma redução de $25,06 \%$ na média geral do peso ao nascer dos cordeiros da restrição pré-natal em relação aos cordeiros sem restrição.

A redução no peso ao nascer dos cordeiros é explicada pelo fato desta variável representar o resultado final do crescimento fetal, sendo este saldo do balanço entre o potencial genético para o crescimento, refletido pela demanda de nutrientes do feto, e os limites impostos ao suprimento destes nutrientes pelo ambiente materno. Assim, durante o terço final da gestação, quando ocorre o maior desenvolvimento do feto, e, consequientemente, a maior demanda por nutrientes, restrições alimentares impostas às ovelhas gestantes limitam a expressão do completo potencial genético para o crescimento do feto (FERREL, 1992).

TABELA 3 - Médias do peso corporal $(\mathrm{kg})$ aos cem dias de gestação e logo após a parição e médias da massa biológica ( $\mathrm{kg}$ cordeiro produzido/kg de ovelha) de ovelhas Santa Inês submetidas ou não a restrição alimentar durante o final da gestação.

\begin{tabular}{lcc}
\hline & Sem restrição & Com restrição \\
\hline Peso aos 100 dias de gestação $(\mathrm{kg})$ & $64,64^{\mathrm{a}}$ & $62,06^{\mathrm{a}}$ \\
Peso após a parição $(\mathrm{kg})$ & $64,55^{\mathrm{a}}$ & $52,03^{\mathrm{b}}$ \\
\hline Massa biológica & $0,12043^{\mathrm{a}}$ & $0,11082^{\mathrm{b}}$ \\
\hline
\end{tabular}

Valores seguidos da mesma letra, na mesma linha, não diferem estatisticamente entre si $(\mathrm{P}<0,01)$ pelo teste de $\mathrm{F}$.

TABELA 4 - Médias do peso ao nascer $(\mathrm{kg})$ de cordeiros Santa Inês machos e fêmeas, oriundos de ovelhas que sofreram ou não restrição alimentar durante o final da gestação.

\begin{tabular}{lc}
\hline \multicolumn{1}{c}{ Fontes de Variação } & Peso ao Nascer $(\mathbf{k g})$ \\
\hline 1.Restrição Pré-Natal & \\
Sem Restrição & $3,830^{\mathrm{a}}$ \\
Com Restrição & $2,870^{\mathrm{b}}$ \\
2.Sexo & \\
Machos & $3,502^{\mathrm{a}}$ \\
Fêmeas & $3,150^{\mathrm{b}}$ \\
3.Interação & \\
Machos - sem restrição & $4,162^{\mathrm{a}}$ \\
Machos - com restrição & $2,893^{\mathrm{b}}$ \\
Fêmeas - sem restrição & $3,474^{\mathrm{a}}$ \\
Fêmeas - com restrição & $2,855^{\mathrm{b}}$ \\
\hline
\end{tabular}

Para cada fonte de variação, valores seguidos da mesma letra, na mesma coluna, não diferem estatisticamente entre si $(\mathrm{P}<0,01)$ pelo teste $\mathrm{F}$.

Ciênc. agrotec., Lavras, v. 30, n. 2, p. 329-334, mar./abr., 2006 
A importância desta variável em termos de produção animal consiste no fato do peso ao nascer estar intimamente correlacionado com a mortalidade perinatal e com o crescimento pós-natal. No Rio Grande do Sul, Oliveira \& Barros (1982) observaram que $80 \%$ dos cordeiros que morreram devido ao complexo inanição/exposição ao ambiente pesavam menos que $3,5 \mathrm{~kg}$ ao nascer. De acordo com o autor, o incremento do peso ao nascer é um objetivo importante a ser considerado no aumento da produtividade da ovinocultura.

A grande redução observada neste trabalho no peso ao nascer dos cordeiros machos $(30,5 \%)$ e fêmeas $(17,8 \%)$ revela a importância da adoção de um nível nutricional adequado para as ovelhas gestantes, principalmente durante o final da gestação. Segundo Kemp et al. (1988), a restrição alimentar imposta durante a gestação pode afetar não só o peso ao nascer dos cordeiros, mas também sua composição corporal e conseqüentemente suas características de carcaça, ressaltando ainda mais a importância da adoção de um nível nutricional adequado para as fêmeas.

A importância da adoção de um nível adequado para as fêmeas no final da gestação fica ainda mais evidente quando consideramos a massa biológica produzida (Tabela 3). A análise deste fator revelou que a restrição alimentar diminuiu a eficiência de produção de cordeiros, resultando uma menor quantidade de produto ( $\mathrm{kg}$ de cordeiro) produzido por $\mathrm{kg}$ de ovelha.

Quanto aos efeitos do sexo sobre o peso ao nascer, os resultados encontrados neste trabalho estão de acordo com os resultados obtidos por Girão et al. (1999) e Pereira et al. (1987), que também encontraram maiores peso ao nascer para cordeiros machos. Por outro lado, Kellaway (1973), estudando os efeitos do plano de nutrição, grupo genético e sexo sobre o crescimento de cordeiros Merinos e cruzados, não verificou diferença no peso ao nascer entre machos e fêmeas nos dois grupamentos genéticos.

\section{CONCLUSÕES}

A restrição nutricional imposta às fêmeas gestantes durante o terço final da gestação afeta o peso ao nascer dos cordeiros, revelando a importância da adoção de um nível nutricional adequado para esta categoria animal.

\section{REFERÊNCIAS BIBLIOGRÁFICAS}

AGRICULTURAL RESEARCH COUNCIL. The nutrient requirements of farm animals. London, 1980. 351 p.
AGRICULTURAL RESEARCH COUNCIL. The nutrient requirements of ruminant livestock. London, 1984. 320 p.

CHARISMIADOU, M. A.; BIZELES, J. A.; ROGDAKIS, E. Metabolic changes during perinatal period in dairy sheep in relation to level of nutrition and breed: I. late pregnancy. Journal Animal Physiologic and Animal Nutrition, [S.1.], v. 84, p. 61-72, 2000.

FERREL, C. L. Nutrient requirements, other factors affect fetal growth. Feedstuffs, [S.1.], v. 17, p. 18-41, 1992.

GIRÃO, R. N.; GIRÃO, E. S.; MEDEIROS, L. P. Desenvolvimento ponderal de cordeiros da raça Santa Inês no Estado do Piauí. In: REUNIÃO ANUAL DA SOCIEDADE BRASILEIRA DE ZOOTECNIA, 36., 1996, Porto Alegre. Anais... Porto Alegre: [s.n.], 1999.

GRANT, A. L.; HELFERICH, W. G. Growth regulation in farm animals. New York: Elsevier, 1991. p. 1-16.

GREENWOOD, P. L.; HUNT, A. S.; HERMANSON, J. W.; BELL, A. W. Effects of birth weight and postnatal nutrition on neonatal sheep: I. body growth and composition, and some aspects of energetic efficiency. Journal Animal Science, Champaign, v. 76, p. 2354-2367, 1998.

HOLST, P. J.; KILLEEN, I. D.; CULLIS, B. R. Nutrition of the pregnant ewe and its effects on gestation length, lamb birth weight and lamb survival. Australian Journal Agricultural Research, Victoria, v. 37, n. 6, p. 647-655, 1986.

IVEY, D. S.; OWENS, F. N.; SAHLU, T.; THE, T. H.; DAWSON, L. J.; CAMPBELL, G. A.; GOETSCH, A. L. Influences of number of fetuses and levels of $\mathrm{CP}$ and $\mathrm{ME}$ in gestation and lactation supplements on performance of Spanish does and kids during suckling and postweaning. Small Ruminant Research, [S.1.], v. 35, p. 123132,2000

KELLAWAY, R. C. The effects of plane of nutrition, genotype and sex on growth, body composition and wool production in grazing sheep. Journal Agricultural Science, Cambridge, v. 80, p. 17-27, 1973. 
KEMP, J. D.; VIMINI, R. J.; ELY, D. G. Influence of maternal frame size and nutritional restriction on growth and development of the postnatal lamb. Journal Animal Science, Champaign, v. 66, p. 3073-3085, 1988.

ELLOR, D. J. Nutritional effects on the fetus and mamary gland during pregnancy. Proceedings Nutrition Society, Cambridge, v. 46, p. 249-257, 1987.

OLIVEIRA, A. C.; BARROS, S. S. Mortalidade perinatal em ovinos no município de Uruguaiana, Rio Grande do Sul. Pesquisa Veterinária Brasileira, [S.1.], v. 2, n. 1, p. 1-7, 1982.

PEREIRA, R. M. A.; LIMA, F. A. M.; FREITAS, J. P.; SILVA, M. A. Fatores ambientais e genéticos como fonte de variação no crescimento de cordeiros da raça Morada Nova, variedade branco, no estado do Ceará. In: REUNIÃO ANUAL DA SOCIEDADE BRASILEIRA DE ZOOTECNIA, 24., 1987, Brasília. Anais... Brasília, DF: SBZ, 1987. p. 321.
ROBINSON, J. J. Pregnancy. In: COOP, I. E. Sheep and goat production. Amsterdam: Elsevier, 1982. p. 103-118.

SIBANDA, L. M.; NDLOVU, L. R.; BRYANT, M. J. Effects of a low plane of nutrition during pregnancy and lactation on the performance of Matebele does and their kids. Small Ruminant Research, [S.1.], v. 32, p. 243-250, 1999.

SIBBALD, A. M.; DAVIDSON, G. C. The effect of nutrition during early life on voluntary food intake by lambs between weaning and 2 years age. Animal Science, Edinburgh, v. 66, p. 697-703, 1998.

SILVEIRA, V. C. P.; LOPEZ, J.; RODRIGUES, F. E. Influência da nutrição materna e do sexo na reserva energética do cordeiro ao nascer. Revista da Sociedade Brasileira de Zootecnia, Viçosa, v. 12, n. 2, p. 242-249, 1992.

WIDDOWSON, E. M.; LISTER, D. Nutritional control of growth. In: PEARSON, A. M.; DUTSON, T. R. Growth regulation in farm animals. London: Elsevier, 1991. p. 67-102. 